\title{
Influencia del área de estudio y el sexo en los estilos de liderazgo
}

Lourdes María Lozano García. Universidad de Granada

Marta Cañadas García. Universidad de Granada

Marina Collado Vicente. Universidad de Granada

\section{Recepción: 24 de mayo de 2017 | Aceptado: 15 de diciembre de 2017 \\ Correspondencia: Lourdes Lozano | Correo-e: Imlg@correo.ugr.es \\ iD 0000-0003-0043-0169}

Citar: Lozano, L., Cañadas, M. y Collado, M. (2017). Influencia del área de estudio y el sexo en los estilos de liderazgo. ReiDoCrea, 6, 356-363.

Resumen: El presente estudio investiga si la interacción entre área de estudio y sexo de los líderes afecta sobre su estilo de liderazgo. Para ello utilizamos el marco teórico de Bass, que diferencia entre liderazgo transformacional y transaccional. Se esperaba que los participantes en áreas características del sexo opuesto mostrasen un estilo de liderazgo distinto al que normalmente se asocia a su sexo. Método: la información se recogió enviando un cuestionario en línea a directores y directoras de departamento de 16 universidades españolas $(\mathrm{N}=137)$, y se analizó mediante pruebas t-test y análisis multivariado de la varianza. Resultados: no se encontraron diferencias significativas entre mujeres y hombres en áreas masculinizadas, por lo que encontramos el efecto esperado en directoras liderando en áreas masculinizadas. Sin embargo, los directores de departamento en áreas feminizadas mantienen la tendencia a un liderazgo transaccional, típicamente asociado a hombres.

Palabras clave: Liderazgo | Sexo

Influence of the field of study and sex in leadership styles

Abstract: This study aims to investigate if the interaction between leaders' field of study and sex influences their leadership styles. We took the approach of Bass' theory of leadership which differentiates between transformational and transactional leadership styles. It was expected that participants in fields characteristic of the opposite sex would show a different leadership style than the one typically associated to their sex. Method: data were collected from the department heads of 16 Spanish universities $(N=137)$ with an online survey, and were analysed using t-tests and multivariate analysis of variance. Results: no significant differences were found between men's and women's leadership style in masculinised fields, which means the hypothesised effect occurs for females in masculinised fields. However, male participants working in feminized fields of study maintained the inclination to a transactional leadership style that is usually associated with the male sex.

Keywords: Leadership | Sex

\section{Introducción}

El liderazgo es un fenómeno que se produce en todos los grupos, tanto de seres humanos como de otros primates (Fernández, Kowalewski, Zunino, 2013; Tecot y Romine, 2012), por lo que su investigación ha suscitado interés a lo largo de la historia. En el campo de la Psicología Social, se empezó a trabajar en liderazgo desde el inicio de la disciplina en el siglo XX (Huici, Molero, Gómez y Morales, 2012), impulsado por los estudios de Lewin y colaboradores en la década de los 40 (Lewin, 1939 citado en Sánchez, 2014). Actualmente, la investigación de este proceso de influencia es de gran importancia en ámbitos organizacionales y relacionados con la Psicología del trabajo y las organizaciones por razones prácticas, ya que si se descubren los factores que caracterizan un estilo de liderazgo eficaz podrían seleccionarse y/o formar a líderes y directivos adecuados, consiguiendo un mejor funcionamiento grupal (Huici et al., 2012). 
La perspectiva del estudio del liderazgo ha ido evolucionando y cambiando desde los años 40 hasta la actualidad, pudiendo diferenciarse cuatro etapas predominantes a lo largo de este periodo: la primera etapa comienza en 1940, enfocada en el rasgo, y el objeto de estudio era la personalidad de los líderes; la segunda etapa, desde 1940 hasta 1960, estaba centrada en los estilos de liderazgo y se estudiaban las conductas de los líderes; en la tercera etapa de 1960 a 1980 los estudios se basaban en la contingencia y el entorno; y por último en la etapa que perdura hasta la actualidad, conocida como la del Nuevo Liderazgo, se estudian variables como el carisma, la capacidad motivadora del líder, consideración individualizada de los seguidores, etc. (Huici et al, 2012). A día de hoy, la conceptualización del liderazgo sigue sin ser clara, ya que éste se relaciona estrechamente con otros conceptos como el poder, la influencia o la autoridad (Moreira, 2010). Bass (1998, en Sánchez 2014) afirma que existen tantas definiciones de liderazgo como personas que han intentado definirlo. Una definición aceptada y que señala un rasgo común a todas las definiciones es la de Hollander (1985, citado en Huici et al, 2012 p.177), según el cual "el liderazgo es un proceso de influencia entre el líder y sus seguidores que tiene por fin conseguir las metas de un grupo, organización o sociedad".

El presente trabajo está basado en el marco teórico de Bass. Este autor construye su enfoque a partir de las teorías propuestas por House (1997, 1978 citado en Solano, Perugini, Benatuil, Nader, y Solano, 2007). Se trata de una de las perspectivas sobre liderazgo que más abarca, tomando en consideración tanto los rasgos y conductas del líder como las variables situacionales. Bass propone tres estilos de liderazgo: transformacional (TRF), transaccional (TRC) y laissez-faire. Según el autor, un líder transformacional promueve el cambio y la innovación en la organización alentando a los seguidores para que logren sus objetivos personales y así llegar a buenos niveles de producción. Actúa a través del carisma, sintetizando la información del medio y promoviendo la cohesión del grupo. Este tipo de liderazgo tiene cuatro dimensiones, que son: carisma, inspiración, estimulación intelectual y consideración individualizada. El carisma es una de las dimensiones más importantes de este tipo de liderazgo y se define como la influencia sobre los demás mediante la creación de una visión, consiguiendo que cambien las metas individuales por las del líder. A veces es difícil separarla de la siguiente dimensión, la inspiración. Ésta hace referencia a cómo el líder transmite a los seguidores su visión de futuro para involucrarlos en el proyecto de cambio. Por otro lado, la estimulación intelectual se da cuando el líder lleva a pensar nuevas soluciones o nuevas maneras de solucionar problemas habituales. Es un componente importante en situaciones críticas. Por último, la consideración individualizada tiene lugar si el líder se ocupa del bienestar de cada uno de los miembros de su equipo, promoviendo protección y cuidado.

El liderazgo transaccional se basa en el intercambio de promesas y favores entre líder y seguidores (Huici et al., 2012). El líder TRC negocia transacciones para conseguir los objetivos de la organización. Éste liderazgo tiene dos dimensiones: recompensa contingente y dirección por excepción. La recompensa contingente es aquella que el líder proporciona al seguidor siempre que este cumpla con los objetivos prefijados, por ejemplo la obtención de un salario. En segundo lugar, la dirección por excepción presenta dos formas, la activa y la pasiva. En la forma activa, el líder hace una monitorización constante para asegurarse de que las actividades se mantienen dentro de las reglamentaciones y procedimientos establecidos. En la forma pasiva, el líder actúa castigando o reforzando un determinado error o acierto que ya ha ocurrido. Por último, el tercer tipo de liderazgo es el laissez faire y consiste en la ausencia de liderazgo, siendo por definición el estilo más ineficaz e inactivo. Implica la ausencia de liderazgo: no se toman decisiones, se demoran las acciones y se ignoran las responsabilidades del líder (Huici et al., 2012). 
Dentro del liderazgo, una línea de investigación cada vez más importante es la que lo relaciona con sexo y género. Con la progresiva incorporación de la mujer a la actividad laboral a lo largo del último siglo y la mencionada importancia que el liderazgo ha ido adquiriendo en las organizaciones y empresas, cada vez se van sumando más investigaciones dirigidas a descubrir la posible influencia del sexo y el género en el comportamiento del líder y los estilos de dirección (W. Orellana, A. Celis, 2011). No obstante, en la mayoría de las sociedades occidentales se observa una escasa ocupación de cargos de dirección por parte de las mujeres; es decir, hombres y mujeres difieren en el acceso a este tipo de puestos. Por ejemplo, en el último estudio del Instituto Nacional de Estadística (2017) sobre la ocupación de las mujeres de altos cargos públicos y privados, el porcentaje de mujeres en el conjunto de Consejos de Administración de las empresas que conforman el lbex-35 era del 19,4\%, y el cargo de mujeres ejercido en los consejos de administración que poseen la presidencia o vicepresidencia es aún más bajo. Esto podría deberse a diferencias en el comportamiento exhibido por los líderes de ambos sexos o a que puedan existir estereotipos que asocian un liderazgo eficaz al ser hombre, lo que convertiría a las mujeres en peores líderes (Moreira, 2010). Este fenómeno parece propio del ser humano, ya que no se da en otro tipo de primates, como demuestra el estudio de Tecot y Romine de 2012, en el que estudiaron el liderazgo en lémures de vientre rojo, y los datos concluyeron que las hembras lideran al grupo más que los machos en diferentes contextos.

En efecto, tradicionalmente se ha asociado el liderazgo a características del género masculino (competitividad, control, autoridad...), lo que hace que características más femeninas como la preocupación por los otros no sean consideradas correctas para puestos de líderes (Cheung y Halpern, 2010; Cuadrado, 2004; Eagly, 1987, citado en Perugini y Solano 2011). A lo largo de la investigación realizada sobre género y liderazgo existen estudios que demuestran que las mujeres tienen un estilo de liderazgo más transformacional y los hombres uno más transaccional, pero también estudios que no han encontrado resultados concluyentes (Del Valle, Santiago y Lugo, 2010). Esta relación entre liderazgo y género ha sido estudiada en diferentes campos, como empresarial, académico, político (Perugini y Solano, 2011) o militar (Moreira, 2010).

El liderazgo no es el único ámbito en el que se observan diferencias entre sexos. Respecto a las diferentes áreas de estudio y conocimiento, también hay estudios que evidencian diferencias significativas entre hombres y mujeres. A principios del siglo $\mathrm{XX}$, encontrar estudiantes universitarias era una tarea difícil. Progresivamente, la mujer ha ido haciéndose hueco en las universidades, constituyendo una mayoría a partir de finales de siglo. Aun así, hoy día sigue habiendo campos en los que las mujeres constituyen una minoría. Existe una persistencia histórica de carreras tradicionalmente masculinizadas o feminizadas (Graña, 2012). En los artículos e investigaciones al respecto se trata de clasificar las diferentes carreras en feminizadas o masculinizadas, dependiendo del número de hombres o mujeres que históricamente ha prevalecido en ellas. Entre ellos encontramos el estudio de Vargas y Requena en Venezuela (2016), en el que se establecen carreras como ingeniería eléctrica, ingeniería mecánica, física y en matemáticas, ingeniería civil y arquitectura como masculinizadas, y biología, odontología, farmacia y psicología como feminizadas. Graña (2012), se refiere a las ingenierías como carreras masculinizadas, y clasifica las carreras de psicología, odontología, ciencias sociales, medicina y química como más propias de mujeres. Incluso dentro de una misma carrera, como en Medicina, hay especialidades muy feminizadas como la Pediatría o Medicina de Familia, y otras áreas claramente dominadas por el sexo masculino como la cirugía y las especialidades vinculadas a la neurología (Gosálbez, Elaso y Merino, 2012). También se pueden apreciar diferencias en las estadísticas estatales sobre datos y cifras del 
sistema universitario español (2014-2015). Con todo esto, en el presente trabajo establecemos que las carreras feminizadas son biología, química, odontología, farmacia, medicina, enfermería, magisterio, psicología, pedagogía, trabajo social, economía, bellas artes, filosofía, filología, geografía e historia. Por otro lado, las carreras masculinizadas son diferentes ingenierías, matemáticas, física y arquitectura.

Un estudio que combinó género y áreas de estudio fue el de Riegle-Crumb, King y Moore en 2016. En dicho estudio se investigó si las mujeres que entran en campos dominados por hombres son más propensas a cambiar de campo que sus compañeras que han elegido otros más feminizados, así como si los hombres que entran en las áreas dominadas por mujeres son más propensos a cambiar de campo que sus compañeros que han elegido un campo más normativo. Los resultados mostraron que se daba mayor abandono en hombres que trabajaban en áreas feminizadas que en mujeres que trabajaban en áreas masculinizadas.

En el presente estudio se analiza cómo se relaciona el estilo de liderazgo con el área de estudio, comparando el estilo de liderazgo de directoras de departamento que trabajan en un área masculinizada con el de compañeras que lo hacen en áreas feminizadas, e igual con la muestra de hombres. El objetivo del estudio es, por tanto, averiguar cómo influye el tipo de área de estudio (más feminizada o más masculinizada) en el estilo de liderazgo de los directores de departamento; es decir, si los jefes de departamento de carreras feminizadas tienen un estilo más propio de mujeres (transformacional) y los jefes de departamento de carreras masculinizadas tienen un estilo transaccional independientemente de su sexo. Se espera que el área de estudio interaccione con el sexo y esto influya sobre el estilo de liderazgo de las directoras y directores de departamento. Si esto es así, deberíamos encontrar que las mujeres pertenecientes a áreas masculinizadas presentan un estilo de liderazgo asociado a hombres (liderazgo TRC), y los hombres en áreas feminizadas muestran un liderazgo más típicamente femenino (liderazgo TRF).

\section{Método}

\section{Participantes}

Como se puede observar en la Tabla 1, la muestra estuvo compuesta por 140 directores de departamento de dieciséis Universidades españolas, de los cuales finalmente participaron ciento treinta y siete debido a falta de información en los cuestionarios administrados. Entre los participantes había 45 mujeres y 92 hombres. De los 92 hombres, 37 eran directores de departamento en áreas de estudio masculinizadas y 55 de áreas de estudio feminizadas. De las 45 mujeres, 15 eran directoras de departamento en áreas de estudio masculinizadas (ingeniería, arquitectura, matemáticas, física y economía) y 30 en áreas de estudio feminizadas (biología, química, ciencias de la salud, ciencias de la educación, psicología, sociología, filosofía, filología, geografía e historia) (véase Tabla 2). 
Tabla 1. Número de participantes de cada Universidad.

\begin{tabular}{ccc} 
Universidad & Frecuencia & Porcentaje \\
\hline UGR & 25 & 18.2 \\
US & 15 & 10.9 \\
UMA & 10 & 7.3 \\
UCO & 10 & 7.3 \\
UAL & 4 & 2.9 \\
UH & 4 & 2.9 \\
UCA & 12 & 8.8 \\
UJA & 2 & 1.5 \\
UAM & 7 & 5.1 \\
UCM & 14 & 10.2 \\
UPM & 1 & .7 \\
URJC & 3 & 2.2 \\
UAH & 3 & 2.2 \\
UCIII & 3 & 2.2 \\
UB & 12 & 8.8 \\
UV & 12 & 8.8 \\
Total & 137 & 100.0
\end{tabular}

Tabla 2. Frecuencias en los dos tipos de Áreas de Estudio.

\begin{tabular}{ccc} 
Tipo de Área & Frecuencia & Porcentaje \\
\hline Masculinizada & 52 & 38 \\
Feminizada & 85 & 62 \\
Total & 137 & 100
\end{tabular}

\section{Instrumentos}

Para medir el estilo de liderazgo se usó el Cuestionario de Estilos de Liderazgo CELID-A (Morales y Molero 1995). El CELID-A está formado por 34 afirmaciones sobre el liderazgo y la acción de liderar autopercibida. Con el fin de facilitar su aplicación se pasó a formato cuestionario online de Google Forms. Para completarlo, los sujetos deben indicar el grado de acuerdo o desacuerdo con cada afirmación mediante una escala Likert de 1-5. A partir de las respuestas se pueden obtener tres tipos de liderazgo, Transformacional (TRF), Transaccional (TRC) o Laissez Faire. El cuestionario también evalúa una serie de subcaracterísticas. Así, el liderazgo TRF tiene cuatro dimensiones: carisma, inspiración, estimulación intelectual y consideración individualizada; y el liderazgo TRC consta de dos dimensiones: recompensa contingente y dirección por excepción. El Laissez Faire hace referencia a la ausencia de liderazgo.

\section{Diseño}

Se utilizó un diseño cuasi experimental $2 \times 2$. Así, el estudio consta de dos variables independientes con dos niveles cada una, el sexo (hombre o mujer) y el área de estudio (carrera masculinizada o carrera feminizada). La variable dependiente es el estilo de liderazgo y tiene tres niveles: transformacional, transaccional, y Laissez Faire.

\section{Procedimiento}

Para llevar a cabo el estudio se envió un correo electrónico a todos los directores de departamento de las áreas de estudio masculinizadas y feminizadas en las Universidades seleccionadas. En él se pedía a cada participante su colaboración y se explicaba lo importante que resultaría para el estudio su participación. En el mismo correo aparecía un enlace al cuestionario online y el plazo de tiempo para completarlo. Para ello, los participantes tenían que evaluar mediante una escala Likert el grado en 
el que cada afirmación se ajustaba al modo de liderar que ellos percibían que desarrollaban.

\section{Resultados}

Una vez recogidos los datos se analizaron utilizando el programa IBM SPSS Statistics. Se realizaron pruebas t-test de muestras relacionadas para cada uno de los 4 grupos, comparando las puntuaciones en Liderazgo Transformacional (TRC) y Liderazgo Transaccional (TRC), obteniendo diferencias significativas en todos los casos como se puede observar en la Tabla 3.

Tabla 3. Resultados de las Pruebas t para cada uno de los grupos, comparando TRF y TRC.

\begin{tabular}{ccccc} 
Grupo & Significación & Resultados & MTRF & MTRC \\
$\begin{array}{c}\text { Mujeres } \\
\text { Área Masculinizada }\end{array}$ & $p=.002$ & TRF $>$ TRC & 3.99 & 3.19 \\
$\begin{array}{c}\text { Hombres } \\
\text { Área Masculinizada } \\
\begin{array}{c}\text { Mujeres } \\
\text { Área Feminizada }\end{array}\end{array}$ & $p<.001$ & TRF $>$ TRC & 3.98 & 2.90 \\
$\begin{array}{c}\text { Hombres } \\
\text { Área Feminizada }\end{array}$ & $p<.001$ & TRF $>$ TRC & 4.07 & 2.86 \\
\hline
\end{tabular}

El TRF es mayor que el TRC para todos los grupos, especialmente para el grupo de mujeres en áreas de estudio feminizadas.

También se realizaron pruebas t-test de muestras independientes para analizar las diferencias entre pares de grupos. A continuación se presenta una tabla (Tabla 4) comparando las puntuaciones en las dimensiones tanto generales como específicas.

Tabla 4. Resultados de las Pruebas t comparando las puntuaciones de los distintos grupos por pares.

\begin{tabular}{|c|c|c|c|}
\hline Grupos & Resultados & MA & $M \mathrm{~B}$ \\
\hline \multirow[t]{2}{*}{$\begin{array}{l}\text { Mujeres Á. Feminizada (A) } \\
\text { Hombres Á. Feminizada (B) }\end{array}$} & $\begin{aligned} \text { TRC: } \mathrm{B}>\mathrm{A} \\
\quad p=.039\end{aligned}$ & 2.86 & 3.12 \\
\hline & $\begin{array}{c}\text { C. Indiv.: } \mathrm{A}>\mathrm{B} \\
\quad p=.01\end{array}$ & 4.52 & 4.27 \\
\hline $\begin{array}{l}\text { Mujeres Á. Feminizada (A) } \\
\text { Hombres Á. Masculinizada (B) }\end{array}$ & $\begin{array}{c}\text { C. Indiv.: } \mathrm{A}>\mathrm{B} \\
\quad p=.004\end{array}$ & 4.52 & 4.14 \\
\hline $\begin{array}{l}\text { Mujeres Á. Feminizada (A) } \\
\text { Mujeres Á. Masculinizada (B) }\end{array}$ & $\begin{array}{c}\text { C. Indiv.: } \mathrm{A}>\mathrm{B} \\
p=.042\end{array}$ & 4.52 & 4.26 \\
\hline $\begin{array}{l}\text { Hombres Á. Masculinizada y } \\
\text { Mujeres Á. Masculinizada }\end{array}$ & \multicolumn{3}{|c|}{ No hay diferencias significativas en ninguna dimensión. } \\
\hline $\begin{array}{l}\text { Hombres Á. Feminizada y } \\
\text { Mujeres Á. Masculinizada }\end{array}$ & \multicolumn{3}{|c|}{ No hay diferencias significativas en ninguna dimensión. } \\
\hline $\begin{array}{c}\text { Hombres Á. Masculinizada y } \\
\text { Hombres Á. Feminizada }\end{array}$ & \multicolumn{3}{|c|}{ No hay diferencias significativas en ninguna dimensión. } \\
\hline
\end{tabular}


Por último, se realizó un análisis multivariante de la varianza (MANOVA). Las pruebas de efectos intersujetos indicaron que hay diferencias significativas con un tamaño del efecto mediano para la dimensión específica Consideración Individualizada $F(.068$, $3)=3.211, p=.025$. Realizando comparaciones múltiples con la prueba Post Hoc de Tukey pudimos comprobar que esta diferencia era significativa únicamente entre hombres en áreas masculinizadas y mujeres en áreas feminizadas.

\section{Discusión}

Los resultados obtenidos indican que la interacción del sexo de los directores/as y el área de estudio a la que pertenecen influye en algunas dimensiones de su estilo de liderazgo. Concretamente, observando los resultados de las pruebas t para cada uno de los grupos que comparan liderazgo TRF y TRC, se puede apreciar que las mujeres directoras de departamento en áreas feminizadas son las que más liderazgo transformacional muestran de los 4 grupos, por lo que parece que el área de estudio ligada a un sexo podría acentuar el estilo de liderazgo típicamente asociado a ese mismo sexo. En el caso de las directoras en áreas típicas del sexo opuesto, no existe ninguna diferencia con los directores en el estilo de liderar, ni en dimensiones generales ni en específicas. Así, las directoras estarían adaptándose al área masculinizada, perdiendo esa consideración individualizada más femenina que comentábamos, mientras que cuando los hombres pertenecen a un área típicamente feminizada, estos siguen mostrando más TRC (estilo típicamente masculino) que las mujeres. También observamos que la dimensión específica consideración individualizada es especialmente alta en las mujeres directoras en áreas feminizadas; sin embargo, los hombres en áreas feminizadas no muestran esta diferencia en dimensión específica. Ésta falta de adaptación por parte de los hombres en áreas dominadas por el sexo opuesto podría explicar los resultados de Riegle-Crumb, King y Moore (2016), que mostraban como la tasa de abandono del puesto de trabajo era mayor en hombres que trabajaban en áreas feminizadas que en mujeres que trabajaban en áreas masculinizadas. Es importante destacar que en áreas masculinizadas no se encontraron diferencias significativas entre el estilo de liderazgo que muestran directores y directoras, para ninguna dimensión.

El presente estudio tuvo varias limitaciones, siendo el tamaño de la muestra probablemente la más importante puesto que de todos los participantes contactados participaron menos de la mitad. Asimismo, los grupos no fueron iguales debido a las diferencias de género que se dan en estos puestos académicos donde independientemente del área, predominan los hombres. No obstante, precisamente porque el número de directoras de departamento en cualquier área es mucho menor que el de directores de departamento, esta desigualdad en la muestra resulta representativa de la población. Por otro lado, el hecho de que el liderazgo transformacional se dé en todos los grupos más que el transaccional podría deberse a que los directores/as de departamento trabajan en el contexto universitario y no un entorno laboral más "ecológico". También podría deberse al efecto de deseabilidad social ya que, gracias a los avances en el estudio del liderazgo y el descubrimiento de las ventajas que un liderazgo más democrático y menos autoritario aporta, las prácticas de liderazgo transformacionales están mejor consideradas.

Además, el cuestionario empleado no está construido específicamente para este contexto, por lo que los participantes pudieron tener dificultades con algunos ítems no aplicables a su trabajo, pero esta era la única opción disponible en español para poder basar la investigación en las teorías más actuales. De igual manera, la literatura reciente sobre la relación entre liderazgo y sexo era escasa por lo que se combinó con literatura sobre liderazgo y género; sin embargo, la variable evaluada en este estudio 
fue sexo, ya que incluir una medida de género incrementaba demasiado el tiempo que requería completar el cuestionario y esto habría reducido aún más la muestra. Por esta razón, se tuvo que asumir que el género y el sexo de los participantes coincidía.

La conclusión que podemos extraer del presente trabajo y los resultados obtenidos es que el sexo no parece determinar el estilo de liderazgo, sino que interactúa con el contexto. De esta manera, al igual que veíamos como en primates no humanos no siempre lideran los machos (Tecot y Romine, 2012), en nuestra sociedad no tendrían por qué liderar solo los hombres, por ser hombres: la cultura influye sobre el estilo de liderar que las personas adoptan y un estilo de liderar eficaz no es una capacidad innata ligada a uno de dos sexos. Por esta razón, es importante seguir estudiando los factores que influyen en el liderazgo, ya que sólo así se podrán descubrir las verdaderas características que hacen de un líder un buen líder.

\section{Referencias}

Caputo, C., Vargas, D., y Requena, J. (2016). Desvanecimiento de la brecha de género en la universidad venezolana. Interciencia, $41(3), 154$.

Datos y Cifras del sistema universitario español - Curso 2014/2015 (2015). MECD: Educación.

Del Valle, V. C., Santiago, J. A. P., y Lugo, M. E. M. (2010). Comparación por género de los estilos de liderazgo en una muestra de gerenciales en Puerto Rico. Revista Puertorriqueña de Psicología, 21, 113-132.

Fernández, V. A., Kowalewski, M., y Zunino, G. E. (2013). Who is coordinating collective movements in black and gold howler monkeys?. Primates, 54(2), 191-199.

Gosálbez, M. I. P., Eraso, Á. B., y Merino, P. P. (2012). Mujeres en sanidad: entre la igualdad y la desigualdad/Women in the health profession: between equality and inequalities. Cuadernos de Relaciones Laborales, 30(2), 497.

Graña, F. (2012). El asalto de las mujeres a las carreras universitarias "masculinas": cambio y continuidad en la discriminación de género. PRAXIS educativa, 12(12), 77-86.

Huici Casal, C., Molero Alonso, F., Gómez Jiménez, Á., y Morales Domínguez, J. F. (2012). Psicología de los grupos.

Instituto Nacional de Estadística (2017). Poder y toma de decisiones. 8.4. Mujeres en altos cargos públicos y privados. España: INE. Recuperado de http://www.ine.es/

Leading Ladies: Leadership of Group Movements in a Pair-Living, Co-Dominant, Monomorphic Primate Across Reproductive Stages and Fruit Availability Seasons. American journal of primatology, 74(7), 591-601.

Lupano Perugini, M. L., y Castro Solano, A. (2011). Teorías implícitas del liderazgo masculino y femenino según ámbito de desempeño. Ciencias Psicológicas, 5(2), 139-150.

Moreira, C. M. (2010). Liderazgo transformacional y género en organizaciones militares. Memoria para optar al grado de Doctor. Universidad Complutense de Madrid.

Orellana Zambrano, W. E., y Celis Ekstrand, A. (2011). Género y capacidades directivas en cooperativas de trabajo asociado. Estudio exploratorio en ctas valencianas. Revista de Estudios Cooperativos (revesco), 2011, num. 105, p. 13-33.

Riegle-Crumb, C., King, B., y Moore, C. (2016). Do They Stay or Do They Go? The Switching Decisions of Individuals Who Enter Gender Atypical College Majors. Sex roles, 74(9-10), 436-449.

Sánchez, J.C. (2012). Psicología de los grupos: teorías, procesos y aplicaciones. McGraw Hill España. Madrid.

Solano, A. C., Perugini, A., Benatuil, M. L., Nader, D., y Solano, M. C. (2007). Teoría y evaluación del liderazgo. Editorial Paidós.

Tecot, S. R., y Romine, N. K. (2012). Leading Ladies: Leadership of Group Movements in a Pair-Living, Co-Dominant, Monomorphic Primate Across Reproductive Stages and Fruit Availability Seasons. American journal of primatology, 74(7), 591-601. 